\title{
Chain of Custody in Forensic Practice: The Need for Standard of Practice Guidelines in Sri Lanka.
}

\author{
Induwara Gooneratne \\ Department of Forensic Medicine, Faculty of Medicine, University of Peradeniya, Sri Lanka
}

This article discusses the notion of 'chain of custody' in forensic practice and its significance in the law of evidence. The need for this discussion arose from the numerous newspaper articles and blogs that highlighted and critiqued handling of forensic samples in Sri Lanka especially related to a recent case that is under investigation.

As the name suggests a chain of custody means the chain/authority under which forensic evidence or a sample has been in custody. Simply put, under whose custody and at which point of time were the forensic samples under consideration. This important concept serves three purposes in evidence law related to criminal investigations. First, it provides details of the official personal under whose hands the samples were and when. It also provides details of the official personal who handled the samples next and so on until it arrives its final destination. In maintaining proper chain of custody, it is a must that all forensic personal properly documents receiving and dispatching of the samples. What I mean by proper documentation is one that is clear, precise, coherent, comprehensive, integral, retrievable and protected. What I mean by it being protected is that the means of record should be kept confidential and protected so that there is minimal or no opportunity for an outsider or an unwanted insider to alter details, mishandle specimen or misplace the register.

The minimum details that should be included in the registry are the case number or reference, sample type, volume/weight, date and time of collection, the person collected, whether the sample is sealed and by whom, date and time of dispatch, the carrier, the place to where the sample was dispatched, reason for dispatching, how it was dispatched, who dispatched it, name, signature, date and time of receiving the sample by the receiving end. One common error that occurs in Sri Lankan labs is that there is only a signature and there is no name when the samples are received. The issue that can arise here, especially when there are short signatures or false signatures by people who receive the samples. Therefore, it is important that both the sender's name with signature and the receiver's name and signatures are clearly stated in the registry. If properly done, this ensures that the sample analysed was actually the sample that was collected. In other words there was no mishandling or contamination of the samples during its course of transport. Of course, this assumption relies heavily on the credibility of the practitioners and the sample handlers in the registry. If all in the chain of custody are to believed then, it can be discerned that the sample was uncontaminated. However this doesn't negate the possibility of others other than the signatories in the registry have access to the samples. This so happens when there are groups of people working together and only one or a few sample storing places are available for example a refrigerator. Further, it is possible that the cleaners or the labourers have access to these facilities. It is up to the forensic unit concerned to develop their own guidelines and standard of practice (SOPs) to uphold the integrity of chain of custody. It is therefore essential that the staff is trained in this regard and that there is some kind of accounting or auditing process in place.

It is seen at many instances in Sri Lanka that the labourers are sent with the samples. And, labourers are utilised to dispatching and receiving of samples. It is always better that people with some training are designated to handle these specimens as they are required to be handled with caution. 
Chain of custody as a legal notion assumes the integrity of the sample obtained initially. If properly maintained it assumes that the sample originally collected is the one that is received by the laboratory for analysis. It further assumes that the sample collected originally was faithful and that the sample received for analysis and being analysed is in its current form and a true representative of the original sample. It is practically possible that the sample is deteriorated or decomposed due to delaying that took place at the original end or due to some issue in collection, store or transport. However unfortunately the status of the sample is not recorded in a routine chain of custody and therefore it is difficult to opine on the integrity of the sample so affected by the environmental insult, improper transport or storage etc. It is the responsibility of each forensic unit to consider these aspects and develop guidelines to collect transport and preserve specimens as necessary.

Most of the guidelines if at all are addressing the external chain of custody. However, it is of paramount importance that a forensic unit with multiple disciplines or with multiple experts develop an internal chain of custody mechanism and a standard of practice. Most forensic units in Sri Lanka do not seem to have either an external or internal standard of practice on the chain of custody. The national level issues raised in courts and media on the internal and external chain of custody of forensic specimen warrants a standard of practice and guidelines in maintaining proper chain of custody to both administer justice and to maintain scientific integrity.

One fact the forensic practitioners ignore is the amount of dismissal of criminal cases based on unavailability of proper evidence. As regard to circumstantial evidence most forensic samples belong, a high degree of credibility is required by the standard of proof in a court room in order to establish the ultimate or penultimate probanda relevant to the case. Therefore a doubtful procedure of chain of custody can easily create a defence and lead to a dismissal of a case.

Another aspect in the chain of custody is the maintenance of either the volumetrics or weights as relevant to the sample. In this way it is possible to examine how much of sample has been taken for analysis and how much then should be left in the sample. It is also possible to audit who has taken the sample for analysis and how much and whether the remaining is kept under optimal conditions if a second opinion or second analysis is ordered or required. Internal guidelines of chain of custody should take this aspect also into consideration.

It is timely that we develop a favorable attitude amongst forensic practitioners regarding the maintenance of both external and internal chain of custody in Sri Lanka. It is also important that we develop knowledge about these aspects among the lawyers and judges especially for them to scrutinize the procedure in their cross examination in court. Training of the people along this most important forensic link is the next important task one needs to look at. Whether the practitioners are trained in maintaining a chain of custody, whether the forensic units have adequate facilities infrastructure and man power, whether the forensic laboratory are periodically checked for protocol awareness or whether there is an internal or external audit on the adherence to the standards of practice protocols and whether the standard of practice guidelines are valid in the eyes of evidence law are a few relevant questions one needs to carefully consider in developing these standard of practice guidelines in maintaining chain of custody in forensic practice. Besides what repercussions are proposed to a practitioner who malpractice accepted protocols should also be given due consideration in formulating guidelines. 